\title{
Efetividade de duas modalidades terapêuticas na redução dos sintomas vocais em pacientes com disfonia comportamental
}

\section{Effectiveness of two therapeutic modalities in the reduction of vocal} symptoms in patients with behavioral dysphonia

\author{
Sauana Alves Leite de Alencar ${ }^{1}$ (D), Larissa Nadjara Alves Almeida ${ }^{1}$ (D), Leonardo Wanderley Lopes $^{2}$ (i), \\ Priscila Oliveira Costa Silva² (1), Anna Alice Almeida² (1)
}

\begin{abstract}
RESUMO
Objetivo: Avaliar a efetividade da terapia de grupo na redução dos sintomas vocais em pacientes com disfonia comportamental e compará-la a uma modalidade de terapia tradicional/individual. Métodos: Trata-se de uma pesquisa explicativa, quantitativa e de intervenção. Participaram 99 pacientes com disfonia comportamental, de ambos os sexos, alocados em dois grupos: Terapia Individual (TI) e Terapia de Grupo (TG). Todos foram submetidos à terapia com abordagem eclética. Foram realizadas oito sessões, sendo a primeira e a última destinadas à avaliação e as outras seis de intervenção. Realizou-se análise estatística descritiva e inferencial para comparar os grupos e os momentos pré e pós-intervenção. Resultados: A maioria dos participantes era do sexo feminino, não usava a voz profissionalmente e com lesão na porção membranosa da prega vocal. Ambos os grupos apresentavam escores médios semelhantes, no início da terapia, fato que mostra a homogeneidade dos grupos. Houve redução de todos os escores da Escala de Sintomas Vocais (ESV) no momento pós-intervenção individual e em grupo. Não foi observada diferença significativa, ao comparar as médias dos escores da ESV pós-terapia entre os grupos. Em relação aos itens da ESV, foi possível observar que alguns podem não detectar as diferenças entre os momentos pré e pós-intervenção. Conclusão: Tanto a TI, quanto a TG foram efetivas na redução significativa dos sintomas vocais. Não houve diferença ao comparar os grupos. Alguns itens da ESV, principalmente no domínio limitação, foram mais sensíveis nos momentos pós-intervenção, em ambas modalidades. O tipo de intervenção influencia a redução dos sintomas vocais.
\end{abstract}

Palavras-chave: Disfonia; Fonoterapia; Prática de grupo; Protocolos; Sinais e sintomas; Voz.

\begin{abstract}
Purpose: To evaluate the effectiveness of two therapeutic approaches in reducing vocal symptoms in patients with behavioral dysphonia. Methods: This was an explanatory, quantitative and interventional study. A total of 99 patients of both sexes with behavioral dysphonia who sought speech therapy participated in this study. These patients were allocated into two groups: individual therapy (IT) and group therapy (GT). All participants were subjected to therapy with an eclectic approach. Eight sessions were conducted, comprising a first and a last session for evaluation, with six intervention sessions in between them. A descriptive and inferential statistical analysis was performed to compare the groups and the moments before and after the intervention. Results: The majority of participants were female, and there was a predominance of patients who did not use their voice professionally and who had a diagnosis of a laryngeal lesion in the membranous portion of the vocal fold. It should be noted that patients presented similar mean scores at the beginning of therapy regardless of the group to which they were allocated, which indicated the homogeneity of the groups. There were reductions in all Vocal Symptoms Scale (VoiSS) scores after individual and group therapy. No significant differences were observed when comparing the mean posttherapy VoiSS scores between the groups in either domain. Some VoiSS items were unable to detect differences between the pre- and posttherapy timepoints. Conclusion: Individual and group therapeutic modalities are effective in significantly reducing self-reported vocal symptoms. The type of intervention influences the reduction in vocal symptoms. Some items of the VoiSS, mainly in the area of limitations, were more sensitive at the posttherapy timepoint in both modalities.
\end{abstract}

Keywords: Dysphonia; Speech therapy; Group practice; Protocols; Signs and symptoms; Voice.

\footnotetext{
Trabalho realizado no Laboratório Integrado de Estudos da Voz - LIEV, Departamento de Fonoaudiologia, Universidade Federal da Paraíba - UFPB - João Pessoa (PB), Brasil.

${ }^{1}$ Departamento de Estatística, Centro de Ciências Exatas e Naturais, Universidade Federal da Paraíba - UFPB - João Pessoa (PB), Brasil.

${ }^{2}$ Departamento de Fonoaudiologia, Centro de Ciências da Saúde, Universidade Federal da Paraíba - UFPB - João Pessoa (PB), Brasil.

Conflito de interesses: Não.

Contribuição dos autores: Todos os autores contribuíram significativamente na construção e desenvolvimento deste trabalho. SALA e LNAA contribuíram com a coleta, tabulação, análise e interpretação dos dados; LWL e POCS participaram da interpretação dos dados e revisão do manuscrito; AAA supervisionou a realização da pesquisa, executou a análise estatística, auxiliou na interpretação dos dados e na revisão do manuscrito.

Financiamento: Este manuscrito está vinculado a um projeto de pesquisa que recebeu financiamento do Conselho Nacional de Desenvolvimento Científico e Tecnológico (CNPq), processo $\mathrm{n}^{\circ}$ 482337/2013-3.

Autor correspondente: Anna Alice Almeida. E-mail: anna alice@uol.com.br.

Recebido: Janeiro 08, 2019; Aceito: Novembro 28, 2019.
} 


\section{INTRODUÇÃO}

A voz é uma função neurofisiológica inata, pois se desenvolve de acordo com a transformação orgânica do indivíduo ${ }^{(1)}$, sob influência de outros aspectos biopsicossociais. É uma ferramenta importante para o processo de comunicação e socialização humana, por enriquecer a transmissão da mensagem articulada, com acréscimo do conteúdo emocional e expressividade ${ }^{(2)}$.

Quando o indivíduo possui alterações ou dificuldade na emissão da voz, diz-se que ele apresenta uma disfonia ${ }^{(3)}$. Existem várias propostas para a classificação das disfonias. A revisão sistemática realizada por Ruotsalainen et al. ${ }^{(4)}$ concluiu que não há classificação universalmente aceita, mas se sabe que a mais difundida, internacionalmente, é a que classifica a disfonia em comportamental e orgânica ${ }^{(5,6)}$.

A disfonia comportamental é caracterizada por alterações vocais relacionadas ao comportamento vocal do sujeito, a partir do uso inadequado da voz ou da exposição a fatores de risco para distúrbios de voz; a orgânica apresenta alterações teciduais ou de estrutura nos órgãos envolvidos na fonação ou em outros sistemas, que impedem a produção natural da voz, independentemente do comportamento vocal do indivíduo ${ }^{(5)}$. Diante disso, os autores constataram que tanto os fatores genéticos, quanto os fatores ambientais auxiliam no surgimento dos problemas de voz e essa interferência pode ser ainda maior quando a pessoa possui algum tipo de ocupação que exige da $\mathrm{vOZ}^{(4,5)}$.

A literatura( ${ }^{(7,8)}$ aponta que as principais formas de manifestação da disfonia são por meio dos sintomas. Os mais frequentes são rouquidão, fadiga vocal, ardor e/ou dor na região da garganta e pescoço, dificuldade em manter a voz, variações na frequência fundamental, falta de volume e projeção vocal, perda na eficiência vocal, pouca resistência ao falar e até perda total da $\mathrm{voz}^{(7)}$.

Estudos evidenciaram que uma forma de tratar os sintomas vocais e a disfonia é a terapia vocal ${ }^{(9,10)}$. A fonoterapia pode ser realizada da forma tradicional, conduzida por fonoaudiólogos com um único paciente ${ }^{(4,10)}$, ou em grupo, que é considerada na literatura como bastante promissora na área de $\mathrm{voz}^{(11-14)}$.

A Fonoaudiologia, em seu processo histórico, se deteve mais à prática terapêutica de caráter individual, que é a mais tradicional e baseada no modelo médico curativo. É um processo que envolve procedimentos fundamentados em uma abordagem mais direta, a partir da execução de exercícios, muitas vezes visando apenas à patologia instalada, visto que, inicialmente, não há observação do comportamento social e emocional do paciente. Por isso, é possível que neste modelo sejam priorizados exercícios vocais e orientações pouco direcionadas às reais condições de vida dos pacientes, no que diz respeito a sua percepção sobre a doença e a interferência dela no cotidiano e na sua inclusão social ${ }^{(12,13,15,16)}$.

Em relação à terapia de grupo, algumas intervenções vêm sendo realizadas, a fim de proporcionar promoção e prevenção da saúde vocal da população, além da reabilitação, quando instalada a disfonia. Esta modalidade terapêutica tem se mostrado efetiva, principalmente em disfonias comportamentais, pois torna possível a observação do comportamento vocal dos participantes em ambiente social, de seus anseios, dúvidas e dificuldades relacionados à terapia vocal, possibilitando a seleção de exercícios e orientações vocais mais direcionadas aos hábitos e queixas vocais, por meio de uma abordagem terapêutica eclética ${ }^{(13,14)}$. Além disso, um paciente poder gerar suporte psicológico aos outros, ao compartilhar sentimentos e vivências positivas e negativas relacionadas ao problema de $\mathrm{voz}^{(11,12)}$.

Assim, é de suma importância desenvolver trabalhos que mostrem evidências científicas em relação a tratamentos para a disfonia, a fim de auxiliar o terapeuta a eleger a melhor abordagem/modalidade para cada paciente, com ganhos direcionados a sua voz e qualidade de vida. Diante disso, o objetivo do estudo foi avaliar a efetividade da terapia de grupo na redução dos sintomas vocais em pacientes com disfonia comportamental, bem como compará-la a uma modalidade de terapia tradicional/individual.

\section{MÉTODOS}

Trata-se de uma pesquisa explicativa, quantitativa, prospectiva e de intervenção. A pesquisa foi submetida ao Comitê de Ética em Pesquisas com Seres Humanos do Centro de Ciências da Saúde da Universidade Federal da Paraíba (UFPB) e aprovada sob protocolo $\mathrm{n}^{\mathrm{o}} 383.061 / 2013$.

\section{População do estudo}

Participaram deste estudo 99 pacientes com disfonia comportamental, de ambos os sexos, que procuraram de forma voluntária terapia fonoaudiológica no serviço de Voz fornecido pelo Laboratório Integrado de Estudos da Voz (LIEV) da UFPB, no âmbito da clínica-escola de Fonoaudiologia, entre fevereiro e dezembro de 2016.

Esses pacientes foram alocados aleatoriamente, pelos pesquisadores, em dois grupos, mediante seleção de prontuários de acordo com a ordem de chegada do paciente no serviço: Terapia Individual (TI) e Terapia de Grupo (TG).

Os critérios de elegibilidade para a participação desta pesquisa foram: ser adulto com diagnóstico de disfonia comportamental, a partir de laudo laringológico e avaliação perceptivo-auditiva realizada por fonoaudiólogo; ter os dados necessários preenchidos pré e pós-intervenção; não ter o número de faltas superior a dois; não ter histórico prévio de tratamento fonoaudiológico para a voz; não ter doença neurológica, genética ou qualquer outra comorbidade que afetasse a cognição, comunicação e voz.

O grupo TG foi constituído, inicialmente, por 94 pacientes, advindos de 15 grupos terapêuticos, com média de seis participantes, cuja terapia ocorreu ao longo do período da pesquisa. Durante a intervenção, houve 24 desistências, restando, assim, 70 indivíduos. Após observação dos critérios de elegibilidade, como ausência do laudo laringológico e número de faltas, foram selecionados 50 participantes. Já o grupo TI, contou, inicialmente, com 65 pacientes, sendo que 16 não atenderam aos critérios de elegibilidade, totalizando, portanto, 49 participantes selecionados (Figuras 1 e 2). Por causa das desistências, não foi possível parear a amostra em relação a sexo, idade e número de participantes em cada grupo, mas, apesar disso, TG e TI tinham características demográficas semelhantes.

Dessa forma, o estudo contou com 99 pacientes com disfonia comportamental, de ambos os sexos, com média de idade de $43( \pm 16,1)$ anos. O grupo Terapia Individual (TI) contou com $49,5 \%(n=49)$ participantes e o grupo Terapia de Grupo (TG) com 50,5\% $(\mathrm{n}=50)$ dos participantes (Tabela 1$)$. 


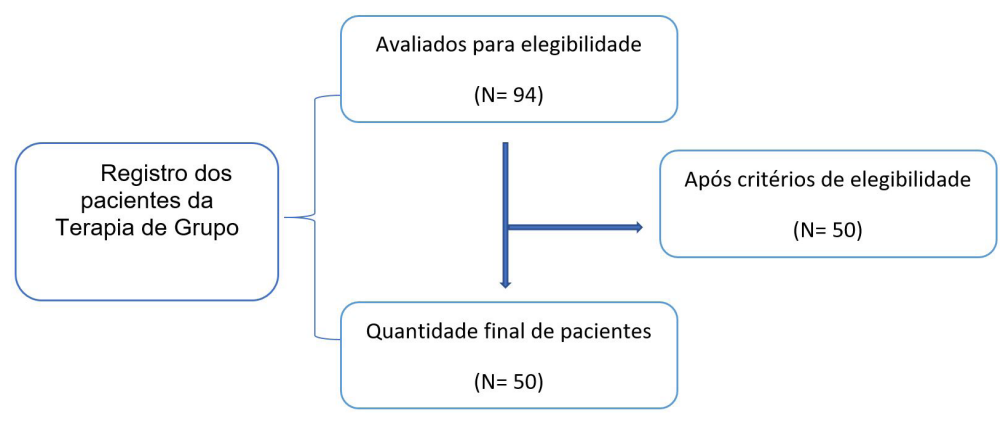

Figura 1. Diagrama representando o fluxo dos participantes da Terapia de Grupo Legenda: $\mathrm{N}=$ número de sujeitos

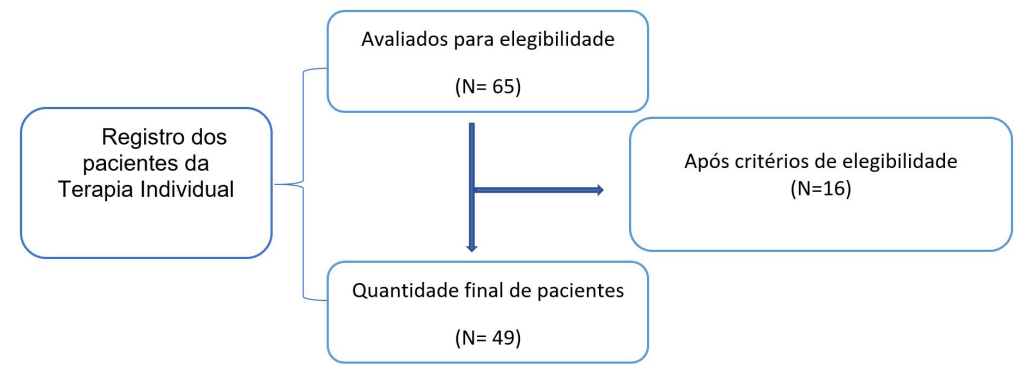

Figura 2. Diagrama representando o fluxo dos participantes da Terapia Individual Legenda: $\mathrm{N}$ = número de sujeitos

Tabela 1. Caracterização da amostra quanto às variáveis demográficas de pacientes com disfonia comportamental, submetidos à terapia de grupo e individual

\begin{tabular}{|c|c|c|c|c|c|c|}
\hline \multirow{2}{*}{ Variável } & \multicolumn{2}{|c|}{ Terapia de Grupo } & \multicolumn{2}{|c|}{ Terapia Individual } & \multicolumn{2}{|c|}{ Total } \\
\hline & $\mathbf{n}$ & $\%$ & $\mathbf{n}$ & $\%$ & $\mathbf{n}$ & $\%$ \\
\hline \multicolumn{7}{|l|}{ Sexo } \\
\hline Mulheres & 36 & 72,0 & 34 & 69,4 & 70 & 70,7 \\
\hline Homens & 14 & 28,0 & 15 & 30,6 & 29 & 29,3 \\
\hline \multicolumn{7}{|l|}{ Uso profissional da voz } \\
\hline Não & 36 & 72,0 & 40 & 81,6 & 76 & 76,8 \\
\hline Sim & 14 & 28,0 & 9 & 18,4 & 23 & 23,2 \\
\hline \multicolumn{7}{|l|}{ Laudo otorrinolaringológico } \\
\hline Lesão na porção membranosa da prega vocal & 14 & 28,0 & 27 & 55,1 & 41 & 41,4 \\
\hline Fenda glótica sem causa orgânica ou neurológica & 10 & 20,0 & 9 & 18,4 & 19 & 19,2 \\
\hline Diagnóstico laríngeo Indefinido & 6 & 12,0 & 5 & 10,3 & 11 & 11,1 \\
\hline Ausência de lesão laríngea & 7 & 14,0 & 7 & 14,3 & 14 & 14,1 \\
\hline Outros & 12 & 24,0 & 2 & 2,0 & 14 & 14,1 \\
\hline
\end{tabular}

Legenda: $\mathrm{n}$ = número de sujeitos. Fonte: João Pessoa, 2016

Foi possível verificar que a maioria dos participantes $(70,7 \% ; n=70)$ era do sexo feminino, sendo $72 \%(n=36)$ na Terapia de Grupo e 69,4\% (n=34) na Terapia Individual. Houve predomínio de pacientes que não usavam a voz profissionalmente ( $72 \%$; $n=36$ na TG e $81,6 \%$; $n=40$ na TI). A maior parte teve diagnóstico laríngeo de lesão na porção membranosa da prega vocal $(41,4 \% ; n=41)$, sendo $28 \%(n=14)$ na Terapia de Grupo de e $55,1 \%(n=27)$ na Terapia Individual.

\section{Coleta de dados}

Foram coletados dados pessoais, como idade, sexo, profissão, grau de escolaridade, avaliação perceptivo-auditiva e laudo laringológico por escrito, realizados, todos, no mesmo serviço.
Utilizou-se a Escala de Sintomas Vocais (ESV) pré e pós-intervenção, para monitorar a autoavaliação dos sintomas vocais.

A ESV é um protocolo que avalia os sintomas vocais apresentados pelo paciente, trazendo informações de funcionalidade, impacto emocional e sintomas físicos que um problema de voz pode acarretar na vida do indivíduo. Contém 30 questões que contemplam três domínios: limitação, físico e emocional. Cada questão tem possibilidade de respostas dentro de uma escala de Likert, que variam de 0 a 4 , de acordo com frequência de ocorrência assinalada: (0) nunca, (1) raramente, (2) às vezes, (3) quase sempre, (4) sempre ${ }^{(17)}$.

O escore limitação equivale às questões $1,2,4,5,6$, $8,9,14,16,17,20,23,24,25$ e 27 . Os itens da subescala emocional são $10,13,15,18,21,28,29$, e 30 e os da subescala física, $3,7,11,12,19,22$ e 26 . A marcação máxima é de 
120 pontos. Os indivíduos com disfonia apresentam escores totais que são superiores a 16 pontos; 11,5 no domínio limitação; 6,5 no físico e 1,5 no emocional. Esses valores são considerados pontos de corte para este instrumento ${ }^{(18)}$.

\section{Procedimentos metodológicos}

O estudo foi iniciado após informar o paciente de todos os procedimentos de pesquisa. Após a resolução de quaisquer perguntas, o Termo de Consentimento Livre e Esclarecido foi assinado para autorizar o início da coleta de dados. Foram realizadas duas modalidades de intervenção: TI e TG.

Ambos os grupos (TI e TG) foram submetidos à terapia com uma abordagem eclética baseada na terapia direta e indireta para a voz. Oito sessões foram realizadas no total, com a primeira e última sessões para avaliação e as demais sessões (segunda a sétima) utilizadas para terapia. Cada sessão de TI durou 30 minutos e foi realizada semanalmente por aproximadamente dois meses.

As sessões TG duraram aproximadamente 90 minutos. Os sujeitos participavam de trocas de experiências e dinâmicas por 60 minutos. Nos 30 minutos restantes, os mesmos exercícios vocais foram realizados como na TI. O programa terapêutico, incluindo os temas e exercícios trabalhados em cada sessão, é delineado no Quadro 1.

\section{Análise de dados}

Realizou-se análise estatística descritiva, que tem como objetivo a descrição dos dados coletados, a fim de verificar a frequência, média e desvio padrão das variáveis estudadas. Posteriormente, foi realizada análise estatística inferencial, com o uso de testes adequados e seguintes objetivos:

- Teste t de Student pareado: comparar os valores médios dos momentos pré e pós-intervenção dos grupos TG e TI;

- Teste t de Student para amostras independentes: comparar as médias dos escores da ESV das modalidades terapêuticas: individual e de grupo;

- Teste Wilcoxon: comparar item a item da ESV pré e pós-intervenção individual e de grupo.

Quadro 1. Descrição das atividades realizadas na terapia individual e de grupo em pacientes com queixas de voz

\begin{tabular}{|c|c|c|}
\hline Sessão & Intervenção & Instrumento \\
\hline 1 & Avaliação & Aplicação da ESV. \\
\hline \multirow{2}{*}{2} & Indireta & $\begin{array}{l}\text { Interação Terapêutica: Dinâmica de apresentação } \\
\text { Aumento de Conhecimento: Anatomofisiologia da produção vocal, voz no ciclo vital }\end{array}$ \\
\hline & Direta & $\begin{array}{l}\text { Intervenção Respiratória: Adequação do tipo respiratório } \\
\text { Suporte Respiratório e Função Vocal: Tempo Máximo de Fonação (TMF) }\end{array}$ \\
\hline \multirow[b]{2}{*}{3} & Indireta & Intervenção Pedagógica; Interação Terapêutica: Mitos e verdades sobre a voz \\
\hline & Direta & $\begin{array}{l}\text { Intervenção Respiratória: Adequação do tipo respiratório } \\
\text { Suporte Respiratório e Função Vocal: TMF } \\
\text { Intervenção - Auditivo; Função vocal; Musculoesquelético; Somatossensorial; Respiratório: Alongamento/relaxamento } \\
\text { da região cervical e cintura escapular; Técnica de fricativos com lateralização de cabeça }\end{array}$ \\
\hline \multirow[b]{2}{*}{4} & Indireta & Intervenção de Aconselhamento; Aumento de Conhecimento: Psicodinâmica vocal; Voz e emoção \\
\hline & Direta & $\begin{array}{l}\text { Intervenção Respiratória: Adequação do tipo respiratório } \\
\text { Suporte Respiratório e Função Vocal: TMF } \\
\text { Intervenção - Auditivo; Função vocal; Musculoesquelético; Somatossensorial; Respiratório: Alongamento/relaxamento } \\
\text { da região cervical e cintura escapular; Técnica de fricativos com lateralização de cabeça; Técnica de trato vocal semiocluído } \\
\text { com tubo de alta resistência }\end{array}$ \\
\hline \multirow[b]{2}{*}{5} & Indireta & Intervenção Pedagógica: Órgãos fonoarticulatórios e Coordenação pneufonoarticulatória \\
\hline & Direta & $\begin{array}{l}\text { Intervenção Respiratória: Adequação do tipo respiratório } \\
\text { Suporte Respiratório e Função Vocal: TMF } \\
\text { Intervenção - Auditivo; Função vocal; Musculoesquelético; Somatossensorial; Respiratório: Alongamento/relaxamento } \\
\text { da região cervical e cintura escapular; Técnica de fricativos com lateralização de cabeça; Técnica de trato vocal semiocluído } \\
\text { com tubo de alta resistência } \\
\text { Musculoesquelético - Manipulação orofacial, Somassensorial: Exercícios miofuncionais para estruturas do sistema } \\
\text { estomatognático }\end{array}$ \\
\hline \multirow[b]{2}{*}{6} & Indireta & Interação Terapêutica; Aumento do Conhecimento: Doenças laríngeas \\
\hline & Direta & $\begin{array}{l}\text { Intervenção Respiratória: Adequação do tipo respiratório } \\
\text { Suporte Respiratório e Função Vocal: TMF } \\
\text { Intervenção - Auditivo; Função vocal; Musculoesquelético; Somatossensorial; Respiratório: Alongamento/relaxamento } \\
\text { da região cervical e cintura escapular; Técnica de fricativos com lateralização de cabeça; Técnica de trato vocal semiocluído } \\
\text { com tubo de alta resistência; Técnica de rotação de língua associado a som nasal } \\
\text { Musculoesquelético - Manipulação orofacial, Somassensorial: Exercícios miofuncionais para estruturas do sistema } \\
\text { estomatognático }\end{array}$ \\
\hline \multirow[b]{2}{*}{7} & Indireta & Intervenção de Aconselhamento; Pedagógica; Interação terapêutica: Comunicação não verbal e expressividade \\
\hline & Direta & $\begin{array}{l}\text { Intervenção Respiratória: Adequação do tipo respiratório } \\
\text { Suporte Respiratório e Função Vocal: TMF } \\
\text { Intervenção - Auditivo; Função vocal; Musculoesquelético; Somatossensorial; Respiratório: Alongamento/relaxamento } \\
\text { da região cervical e cintura escapular; Técnica de fricativos com lateralização de cabeça; Técnica de trato vocal semiocluído } \\
\text { com tubo de alta resistência; Técnica de rotação de língua associado a som nasal } \\
\text { Musculoesquelético - Manipulação orofacial, Somassensorial: Exercícios miofuncionais para estruturas do sistema } \\
\text { estomatognático; Técnica de sobrearticulação }\end{array}$ \\
\hline 8 & Reavaliação & Aplicação da ESV. \\
\hline
\end{tabular}

Legenda: ESV = Escala de Sintomas Vocais; TMF = Tempo Maximo de Fonação 
É importante mencionar que, para classificar os diagnósticos laríngeos, os pacientes foram alocados em quatro categorias: ausência de lesão laríngea, fenda glótica sem causa orgânica ou neurológica, lesão na porção membranosa das pregas vocais (nódulos, pólipos e cistos) ${ }^{(19)}$ e diagnóstico laríngeo indefinido. Ressalta-se que todos eles tinham alteração de voz, conforme avaliação perceptivo-auditiva realizada por fonoaudiólogo especialista em voz e com envolvimento comportamental.

As diferenças foram consideradas significativas quando resultaram em $\mathrm{p}<0,05$. A análise estatística foi realizada por meio do software Statistical Package for Social Sciences (SPSS), versão 20.0.

\section{RESULTADOS}

A comparação das médias dos escores da ESV, nos momentos pré e pós-intervenção, em ambos os grupos, observou-se que, na terapia de grupo, houve a redução de todos os domínios da ESV (Tabela 2). A média do escore total passou de 45,56 $( \pm 23,10)$ para 32,20 $( \pm 18,85)(\mathrm{p}<0,0001)$, o domínio limitação, de 25,77 $( \pm 13,67)$ para $18,64( \pm 11,48)(\mathrm{p}<0,0001)$, o emocional, de $8,46( \pm 8,15)$ diminuiu para $4,88( \pm 5,87)(\mathrm{p}<0,0001)$ e o físico, de 11,36 $( \pm 4,95)$ para 8,68 $( \pm 4,21)(\mathrm{p}<0,0001)$. Já na terapia individual, percebeu-se redução significativa na média do escore total da ESV, que passou de 47,85 $( \pm 27,21)$ para $36,12( \pm 24,26)(\mathrm{p}=0,001)$ o domínio limitação, de 27,67 $( \pm 16,09)$ para 21,20 $( \pm 14,32)(\mathrm{p}=0,002)$, o emocional, de $9,32( \pm 9,13)$ para $6,85( \pm 7,45)(\mathrm{p}=0,025)$ e o físico, de $10,85( \pm 6,60)$ para $8,06( \pm 5,77)(\mathrm{p}=0,004)$. Desta forma, foi possível observar que os pacientes submetidos à terapia individual também tiveram redução significativa de seus sintomas vocais.

É importante citar que foi realizada a comparação das médias dos escores da ESV intergrupos, TG e TI. Destaca-se que os pacientes apresentavam escores semelhantes no início da terapia, independentemente do grupo em que foram alocados, fato que mostra a homogeneidade dos grupos. Não foi observada diferença significativa ao comparar as médias dos escores da ESV entre os grupos, em nenhum dos domínios. Estes dados comprovam que as sessões terapêuticas, tanto no grupo TI, quanto no TG foram efetivas na diminuição dos sintomas vocais, observada na redução das médias pré e pós-intervenção.

A Tabela 3 contém dados sobre a associação da frequência de aumento e redução dos escores nos domínios da ESV com os momentos pré e pós-intervenção de grupo e individual. Observou-se que, apesar de ambas as modalidades serem efetivas, a redução dos sintomas esteve associada ao tipo de terapia ao qual o indivíduo foi submetido e foi significativamente maior na TG, nos domínios total ( $p=0,049)$, em que 54,9\% $(n=45)$ dos voluntários melhoraram, emocional $(\mathrm{p}=0,002)$, em que $60,9 \%$

Tabela 2. Comparação das médias dos domínios da Escala de Sintomas Vocais nos momentos pré e pós-terapia de grupo e individual em indivíduos com disfonia comportamental

\begin{tabular}{|c|c|c|c|c|c|}
\hline \multirow{2}{*}{ Variável } & \multicolumn{2}{|c|}{ Pré-terapia } & \multicolumn{2}{|c|}{ Pós-terapia } & \multirow{2}{*}{ valor de $p$} \\
\hline & Média & Desvio Padrão & Média & Desvio Padrão & \\
\hline ESV- T (TG) & 45,56 & 23,10 & 32,20 & 18,85 & $0,0001^{*}$ \\
\hline ESV - L (TG) & 25,77 & 13,67 & 18,64 & 11,48 & $0,0001^{*}$ \\
\hline ESV- E (TG) & 8,46 & 8,15 & 4,88 & 5,87 & $0,0001^{*}$ \\
\hline ESV - F (TG) & 11,36 & 4,95 & 8,68 & 4,21 & $0,0001^{*}$ \\
\hline ESV-T (TI) & 47,85 & 27,21 & 36,12 & 24,26 & $0,001^{*}$ \\
\hline ESV- L (TI) & 27,67 & 16,09 & 21,20 & 14,32 & $0,002^{*}$ \\
\hline ESV- E (TI) & 9,32 & 9,13 & 6,85 & 7,45 & $0,025^{*}$ \\
\hline ESV- F (TI) & 10,85 & 6,60 & 8,06 & 5,77 & $0,004^{*}$ \\
\hline
\end{tabular}

Teste t de Student pareado; * significância $p<0,05$

Legenda: TG = Terapia de grupo; TI = Terapia individual; ESV - T = Escala de Sintomas Vocais domínio total; ESV - L = Escala de Sintomas Vocais domínio limitação; ESV - E = Escala de Sintomas Vocais domínio emocional; ESV - F = Escala de Sintomas Vocais domínio físico

Tabela 3. Associação entre redução e aumento dos escores dos domínios da Escala de Sintomas Vocais com o tipo de terapia a qual o indivíduo foi submetido

\begin{tabular}{|c|c|c|c|c|c|c|}
\hline \multirow{2}{*}{\multicolumn{2}{|c|}{ Variáveis }} & \multicolumn{2}{|c|}{ Terapia de Grupo } & \multicolumn{2}{|c|}{ Terapia Individual } & \multirow{2}{*}{ valor de $p$} \\
\hline & & $n$ & $\%$ & $\mathrm{n}$ & $\%$ & \\
\hline \multicolumn{7}{|l|}{ ESV-T PÓS } \\
\hline & Redução & 45 & 54,9 & 37 & 45,1 & $0,049^{*}$ \\
\hline & Aumento & 5 & 29,4 & 12 & 70,6 & \\
\hline \multicolumn{7}{|l|}{ ESV-E PÓS } \\
\hline & Redução & 42 & 60,9 & 27 & 39,1 & $0,002^{*}$ \\
\hline & Aumento & 8 & 26,7 & 22 & 49,5 & \\
\hline \multicolumn{7}{|l|}{ ESV-F PÓS } \\
\hline & Redução & 44 & 57,9 & 32 & 42,1 & $0,007^{*}$ \\
\hline & Aumento & 6 & 26,1 & 17 & 73,9 & \\
\hline
\end{tabular}

Teste Qui-quadrado; * significância $p<0,05$

Legenda: $\mathrm{n}$ = número de sujeitos; ESV - T = Escala de Sintomas Vocais domínio total; ESV - E = Escala de Sintomas Vocais domínio emocional; ESV - F = Escala de Sintomas Vocais domínio físico; Redução = Redução do escore da ESV; Aumento = Aumento dos escores da ESV 
$(\mathrm{n}=42)$ apresentaram escores reduzidos no momento pós, e no físico $(p=0,007)$, com 57,9\% $(n=44)$ escores reduzidos, em relação ao momento pré-terapia, ou seja, a terapia de grupo se destacou na redução dos sintomas vocais totais, emocionais e físicos. A redução ou aumento dos escores do domínio limitação não esteve associada ao tipo de terapia $(p=0,142)$ (Tabela 3$)$.

Em relação à modificação dos itens da ESV pela comparação das respostas dadas pelos pacientes, nos momentos pré e pós-intervenção de grupo, os itens que apresentaram mudanças significativas no momento pós-intervenção foram: 02, 03, 04, $07,08,11,15,16,17,18,19,20,21,22,23,24,25,28$ e 29. Sendo assim, 63,4\% $(n=19)$ dos itens da ESV modificaram pós-intervenção de grupo e 36,6\% $(n=11)$ não apresentaram modificações (Tabela 4).

A Tabela 5 mostra a modificação dos itens da Escala de Sintomas Vocais pela comparação das respostas da terapia individual, os itens que apresentaram mudanças significativas foram: $04,10,11,12,13,14,15,17,19,20,23,24,25$ e 29. Assim, 46,6\% $(\mathrm{n}=14)$ de itens da ESV modificaram no momento pós-terapia individual e 53,4\% $(n=16)$ não apresentaram mudanças. Em todas essas questões, as respostas no momento pós-intervenção tiveram um número igual ou significativamente inferior às do momento pré-intervenção, entre os pacientes com disfonia comportamental avaliados (Tabela 5).

Tabela 4. Análise da modificação dos itens da Escala de Sintomas Vocais pela comparação das respostas pré e pós-terapia de grupo

\begin{tabular}{|c|c|c|c|c|c|}
\hline & ITENS DA ESV & $\begin{array}{c}\text { Pós<Pré } \\
\text { (n) }\end{array}$ & $\begin{array}{c}\text { Pós>Pré } \\
\text { (n) }\end{array}$ & $\begin{array}{c}\text { Pós=Pré } \\
\text { (n) }\end{array}$ & valor de $p$ \\
\hline 02 & Você tem dificuldades para cantar? & 25 & 4 & 21 & $0,0001^{*}$ \\
\hline 03 & Sua garganta dói? & 17 & 8 & 25 & $0,036^{*}$ \\
\hline 04 & Sua voz é rouca? & 24 & 7 & 19 & $0,001^{*}$ \\
\hline 07 & Você tosse ou pigarreia? & 24 & 10 & 16 & $0,006^{*}$ \\
\hline 08 & Sua voz é fraca/baixa? & 21 & 10 & 19 & $0,007^{*}$ \\
\hline 11 & Você sente alguma coisa parada na garganta? & 22 & 8 & 20 & $0,025^{*}$ \\
\hline 15 & Seu problema de voz deixa você estressado ou nervoso? & 17 & 7 & 26 & $0,014^{*}$ \\
\hline 16 & Você tem dificuldade para falar em locais barulhentos? & 22 & 8 & 20 & $0,021^{*}$ \\
\hline 17 & É difícil falar forte (alto) ou gritar? & 25 & 5 & 20 & $0,001^{*}$ \\
\hline 18 & O seu problema de voz incomoda sua família ou amigos? & 16 & 9 & 25 & $0,047^{*}$ \\
\hline 19 & Você tem muita secreção ou pigarro na garganta? & 21 & 13 & 16 & $0,037^{*}$ \\
\hline 20 & O som da sua voz muda durante o dia? & 23 & 6 & 21 & $0,008^{*}$ \\
\hline 21 & As pessoas parecem se irritar com sua voz? & 17 & 12 & 31 & $0,003^{*}$ \\
\hline 22 & Você tem o nariz entupido? & 20 & 8 & 22 & $0,028^{*}$ \\
\hline 23 & As pessoas perguntam o que você tem na voz? & 20 & 7 & 23 & $0,008^{*}$ \\
\hline 24 & Sua voz parece rouca e seca? & 20 & 8 & 22 & $0,020^{*}$ \\
\hline 25 & Você tem que fazer força para falar? & 25 & 6 & 19 & $0,001^{*}$ \\
\hline 28 & Sua voz faz você se sentir incompetente? & 18 & 3 & 29 & $0,007^{*}$ \\
\hline 29 & Você tem vergonha do seu problema de voz? & 17 & 3 & 30 & $0,0001^{*}$ \\
\hline
\end{tabular}

Teste de Wilcoxon (teste dos sinais) para comparação de variáveis ordinais; * Significância de $p<0,05$

Legenda: $\mathrm{n}=$ número de sujeitos; ESV = Escala de Sintomas Vocais

Tabela 5. Análise da modificação dos itens da Escala de Sintomas Vocais pela comparação das respostas pré e pós-terapia individual

\begin{tabular}{|c|c|c|c|c|c|}
\hline & ITENS DA ESV & $\begin{array}{c}\text { Pós<Pré } \\
\text { (n) }\end{array}$ & $\begin{array}{c}\text { Pós>Pré } \\
\text { (n) }\end{array}$ & $\begin{array}{c}\text { Pós=Pré } \\
\text { (n) }\end{array}$ & valor de $p$ \\
\hline 04 & Sua voz é rouca? & 23 & 4 & 28 & $0,001^{*}$ \\
\hline 10 & Você se sente mal ou deprimido por causa do seu problema de voz? & 16 & 5 & 28 & $0,042^{*}$ \\
\hline 11 & Você sente alguma coisa parada na garganta? & 20 & 8 & 21 & $0,012^{*}$ \\
\hline 12 & Você tem nódulos inchados (íngua) no pescoço? & 16 & 8 & 25 & $0,016^{*}$ \\
\hline 13 & Você se sente constrangido por causa do seu problema de voz? & 19 & 6 & 24 & $0,011^{*}$ \\
\hline 14 & Você se cansa para falar? & 26 & 12 & 11 & $0,011^{*}$ \\
\hline 15 & Seu problema de voz deixa você estressado ou nervoso? & 23 & 7 & 19 & $0,004^{*}$ \\
\hline 17 & É difícil falar forte (alto) ou gritar? & 20 & 6 & 23 & $0,006^{*}$ \\
\hline 19 & Você tem muita secreção ou pigarro na garganta? & 24 & 10 & 15 & $0,023^{*}$ \\
\hline 20 & O som da sua voz muda durante o dia? & 21 & 7 & 21 & $0,003^{*}$ \\
\hline 23 & As pessoas perguntam o que você tem na voz? & 22 & 5 & 22 & $0,0001^{*}$ \\
\hline 24 & Sua voz parece rouca e seca? & 21 & 8 & 20 & $0,002^{*}$ \\
\hline 25 & Você tem que fazer força para falar? & 24 & 9 & 16 & $0,019^{*}$ \\
\hline 29 & Você tem vergonha do seu problema de voz? & 16 & 10 & 23 & $0,044^{*}$ \\
\hline
\end{tabular}

Teste de Wilcoxon (teste dos sinais) para comparação de variáveis ordinais; *Significância de $p<0,05$

Legenda: $\mathrm{n}=$ número de sujeitos; ESV = Escala de Sintomas Vocais 


\section{DISCUSSÃO}

Este estudo versou sobre dados de terapia fonoaudiológica em duas modalidades: individual (TI) e de grupo (TG). Ambas tiveram abordagem eclética e mostraram efetividade na redução dos sintomas vocais para a população com disfonia comportamental. Estudos trazem evidências de que a terapia individual é efetiva na redução dos sintomas vocais ${ }^{(14,20)}$. Então, foi importante pesquisar a efetividade da terapia de grupo sobre esse aspecto, a fim de respaldar práticas grupais em serviços de saúde que atendem altas demandas.

A Fonoaudiologia dispõe de três tipos de abordagens de intervenção: a terapia direta, que abrange os exercícios vocais, a indireta, que trabalha com aconselhamentos e orientações sobre os cuidados com a higiene vocal e a eclética, que utiliza uma combinação de estratégias de abordagens diretas e indiretas ${ }^{(4)}$. Todas elas buscam o mesmo objetivo de reduzir as alterações vocais e os impactos profissionais, sociais e emocionais resultantes da disfonia.

Atualmente, os fonoaudiólogos utilizam ambas as abordagens e criam um programa de tratamento customizado, ou terapia vocal eclética ${ }^{(11)}$. Ruotsalainen et al. ${ }^{(4)}$ categorizaram intervenções para tratar disfonias comportamentais em técnicas de tratamento diretas e indiretas. Na utilização de técnicas diretas, a intervenção é focada no aparelho fonador, trabalhando com os componentes da produção vocal propriamente dita, como respiração e configuração laríngea. Já as técnicas indiretas, partem do pressuposto de que uma abordagem educacional ajuda o indivíduo a identificar os fatores mantenedores do problema e, assim, levar à conscientização e mudança no comportamento. A terapia indireta se concentra na eliminação de fatores mantenedores da alteração vocal. Neste tipo de tratamento, a intervenção é focada na modificação de funções mentais ou corporais que influenciam na produção da voz. O estudo menciona a possibilidade de combinação das duas abordagens, por meio de educação em saúde voltada para a voz, somada a técnicas focadas na qualidade vocal.

Em relação à população da pesquisa, ambos os grupos foram formados por maioria do sexo feminino, não profissionais da voz, com diagnóstico de lesão na porção membranosa de pregas vocais ou fenda glótica.

Estudos mostraram que as mulheres têm mais distúrbios da voz do que os homens, pois apresentam duas vezes mais probabilidade de desenvolver problemas vocais, devido a questões anatomofisiológicas da sua configuração laríngea ${ }^{(21,22)}$. Além disso, as mulheres procuram mais os serviços de saúde do que homens e representam até $76 \%$ dos encaminhamentos clínicos para área de $\mathrm{voz}^{(23)}$.

Percebeu-se que houve maior procura de pacientes que não utilizavam a voz como meio de trabalho, fato que pode constatar que os sintomas vocais estão presentes em diferentes populações, apesar de saber-se que pessoas com profissões de grande exigência vocal correm mais o risco de desenvolver problemas de voz. Os dados mostraram que a preocupação e queixas relacionadas à voz vão além de tê-la como instrumento de trabalho. A procura pela reabilitação vocal está relacionada à intensidade com que o problema afeta a qualidade de vida e as tarefas cotidianas ${ }^{(19)}$ e não necessariamente a profissão exercida.

De forma geral, existem diversos fatores que contribuem para o aparecimento de sintomas e alterações vocais, fato este que deixa claro que a disfonia não pode ser explicada por uma única causa ${ }^{(24)}$. O aparecimento de disfonia encontra-se relacionado a inúmeros fatores etiológicos, comuns entre a população geral. O tipo de personalidade, o estilo de vida e os hábitos vocais podem contribuir para um padrão saudável, ou não, de produção vocal ${ }^{(25)}$. Estas situações podem aumentar o risco de aparecimento de disfonia.

A maioria dos pacientes da pesquisa apresenta lesão na porção membranosa da prega vocal. As disfonias comportamentais contemplam essas lesões, além das fendas glóticas, ou, ainda, alteração na voz com ausência de modificação laríngea. O surgimento desse tipo de disfonia está associado ao uso incorreto ou abusivo da voz, que pode gerar fonotraumas repetitivos, contribuindo para a instalação das lesões laríngeas. Este é o diagnóstico mais comumente encontrado na população de disfônicos ${ }^{(26)}$.

As lesões benignas mais comuns são nódulo e pólipo vocal, granuloma, edema de Reinke e leucoplasias, sendo o nódulo a lesão mais incidente em mulheres e mais frequente na prática clínica. A terapia fonoaudiológica é considerada efetiva no tratamento dessas alterações vocais ${ }^{(4,8)}$.

A terapia de grupo foi efetiva na redução dos sintomas vocais em todos os domínios da ESV, assim como a individual. Foi possível observar que, no momento pós-intervenção de grupo, apesar de ser uma modalidade terapêutica mais recente, mostrou-se excelente estratégia, sobretudo em serviços públicos, onde existe grande demanda para atendimento fonoaudiológico.

Almeida et al. ${ }^{(13)}$ realizaram revisão sistemática da literatura, a fim de verificar os efeitos da fonoterapia de grupo em pacientes com disfonia. Como resultado, pôde-se observar que a terapia vocal de grupo tem sido descrita como modalidade terapêutica efetiva para a disfonia, em todas as fases do ciclo vital, tanto em ações de promoção à saúde vocal, quanto na prevenção e reabilitação de distúrbios de voz.

Em relação à terapia individual, estudos de intervenção fonoaudiológica para $\mathrm{Voz}^{(6,10)}$ demonstraram a sua efetividade e explicaram que se trata de um processo que envolve procedimentos de diversas naturezas, a fim de desenvolver a melhor comunicação oral, com redução do esforço fonatório, diminuição dos sintomas vocais e adequação da qualidade vocal e dos parâmetros acústicos da voz, de acordo com as necessidades pessoais, sociais e profissionais do indivíduo.

Ribeiro et al. ${ }^{(27)}$ realizaram um estudo retrospectivo, com revisão de 42 prontuários de mulheres portadoras de disfonia comportamental, com objetivo de avaliar os resultados das avaliações vocais, laríngeas e de autopercepção após o tratamento fonoaudiológico. Foram utilizadas terapia direta, com a realização de técnicas vocais, e indireta, com orientação sobre cuidados com a saúde vocal. Observou-se, após as sessões de terapia, melhora dos parâmetros perceptivo-auditivos de grau geral da disfonia e rugosidade, nas medidas acústicas de jitter e shimmer, bem como melhora da imagem laríngea e impacto positivo da voz na qualidade de vida das professoras avaliadas após o tratamento fonoaudiológico. Pode-se constatar, de acordo com a literatura e com este estudo, que a terapia eclética traz benefícios nos aspectos multidimensionais da voz.

Quando comparadas as duas modalidades, os grupos se comportaram de forma semelhante, nos momentos pré e pós-intervenção, visto que o comparativo intergrupo de terapia individual e de grupo não foi significativo. Este fato leva a crer que ambas foram efetivas e com ganhos importantes na redução dos sintomas vocais, o que confirma e acrescenta aos dados da literatura. 
Um estudo realizou a apresentação do Programa Integral de Reabilitação Vocal (PIRV), que utilizou uma abordagem eclética ao associar técnicas vocais a conhecimentos de higiene vocal e atitude comunicativa ${ }^{(10)}$. A proposta do programa inclui seis sessões iniciais, explorando aspectos inicialmente apresentados na abordagem global nas disfonias. Após seis sessões terapêuticas, os autores puderam perceber a melhora da qualidade vocal, padrão laríngeo, além de valores mais satisfatórios no Índice de Desvantagem Vocal e Questionário de Qualidade de Vida em Voz ${ }^{(28)}$.

Em relação à evolução clínica, a terapia de grupo parece ser promissora ${ }^{(13)}$. Vários estudos evidenciaram a efetividade desta modalidade terapêutica utilizando a abordagem eclética em diversos aspectos vocais, como sintomas proprioceptivos e auditivos ${ }^{(22)}$, redução dos fatores de risco vocais ${ }^{(14)}$, além do aumento das estratégias de enfrentamento na disfonia. Esses estudos citados utilizaram, inclusive, o mesmo programa terapêutico em grupo aplicado neste estudo.

Observou-se, ainda, com os dados da pesquisa, que o tipo de modalidade terapêutica influenciou significativamente a redução dos sintomas vocais nos domínios total, emocional e físico, principalmente nos pacientes da terapia de grupo.

Como a terapia de grupo é efetiva no tratamento da disfonia, já era esperado que os sintomas reduzissem de modo geral, mas o fato de esta redução se destacar em relação a dos aspectos emocionais relativos à disfonia pode ser reflexo do favorecimento de uma melhor relação pessoal e interpessoal no grupo, que pode proporcionar um ambiente mais satisfatório de enfrentamento por meio do compartilhamento, da troca de experiência, além de auxiliar no processo de reabilitação do paciente nos aspectos físico, social e emocional ${ }^{(13)}$.

Em relação aos itens da ESV, foi possível observar que alguns podem não detectar as diferenças entre os momentos pré e pós-intervenção. Por isso, é importante que outros estudos sejam realizados utilizando esse protocolo, a fim de buscar mais informações, no que se refere à sensibilidade dos itens.

Os itens que foram mais sensíveis à melhora, em ambas as modalidades, pertencem ao domínio limitação, que contempla sintomas relacionados à funcionalidade, ou seja, as limitações do paciente em relação à produção da voz causada pela disfonia ${ }^{(17)}$. Neste domínio, são contemplados os sintomas como rouquidão, perda da voz, voz fraca/baixa, falhas na voz e cansaço ao falar. A literatura apontou que existe correlação positiva entre o domínio limitação da ESV e a intensidade do desvio vocal, ou seja, pacientes com maior intensidade do desvio vocal podem apresentar maior número de sintomas vocais relacionados à limitação ${ }^{(29)}$.

O grupo favorece uma atmosfera mais natural da comunicação do cotidiano, facilita o aprendizado das habilidades motoras das técnicas que podem ser mais efetivas do que apenas na presença do fonoaudiólogo, ajuda o indivíduo a enfrentar a doença e diminuir níveis de ansiedade ${ }^{(13)}$, além de um paciente poder gerar suporte psicológico aos outros, pois permite que os participantes interajam, a partir do compartilhamento de experiências e conhecimentos, o que proporciona o desenvolvimento de uma nova visão de si e do outro diante da doença, diminuindo a ansiedade gerada, direcionando o sujeito a enfrentar melhor o estressor ${ }^{(14)}$. O convívio proporcionado pelo grupo favorece a formação de vínculos terapeuta-paciente e dos pacientes entre si, tornando o ambiente mais acolhedor e motivador para participação e melhor adesão à proposta terapêutica ${ }^{(29)}$.
A literatura comprova a efetividade da terapia de grupo, tanto na prevenção das alterações vocais, como na melhoria da qualidade de vida, exposição a fatores de risco e sintomas vocais, redução do índice de desvantagem vocal, medidas acústicas e perceptivo-auditivas, durante a reabilitação ${ }^{(5,13,14)}$. Além disso, como já foi mencionado, o grupo gera um ambiente capaz de abranger questões biopsicossociais dos participantes, de modo que o tratamento seja verdadeiramente multidimensional ${ }^{(12)}$.

Diante dos resultados da pesquisa, observa-se que a terapia de grupo se sobressaiu, em relação à individual, na redução dos sintomas vocais, sendo possível afirmar que o ambiente terapêutico de grupo é altamente propício para o tratamento da voz. Este achado é importante, pois contribui cientificamente e na prática clínica de fonoaudiólogos inseridos em serviços com alta demanda, possibilitando a prática de processos grupais na reabilitação de pacientes com disfonia comportamental, com a finalidade de reduzir as filas de espera. Além disso, o presente estudo permite a replicabilidade de sua metodologia nesses serviços.

\section{CONCLUSÃO}

Tanto a terapia de grupo, quanto a terapia tradicional/individual foram efetivas na redução significativa dos sintomas vocais autorreferidos. Não houve diferença significativa ao comparar os grupos.

Foi possível observar que alguns itens da ESV, principalmente no domínio limitação, foram os mais sensíveis nos momentos pós-terapia, em ambas as modalidades. Observou-se que a terapia de grupo se sobressaiu em relação à individual, na redução de mais sintomas listados na ESV.

\section{REFERÊNCIAS}

1. Maia AA, Michalick-Triginelli MF. Relação entre transtorno do défict de atenção/hiperabilidade, dinânica familiar, disfonia e nódulo vocal em crianças. Rev Cienc Méd. 2012;15(5):379-89.

2. Souza OC, Hanayama EM. Fatores psicológicos relacionados adisfonia funcional e a nódulos vocais em adultos. Rev CEFAC. 2005;7(3):38897.

3. Behlau M. O livro do especialista. Rio de Janeiro: Revinter; 2008. Vol. 1.

4. Ruotsalainen J, Sellman J, Lic P, Lehto L, Verbeek J. Systematic review of the treatment of functional dysphonia and prevention of voice disorders. Otolaryngol Head Neck Surg. 2008;138(5):557-65. http://dx.doi.org/10.1016/j.otohns.2008.01.014. PMid:18439458.

5. Simberg S, Santtila P, Soveri A, Varjonen M, Sala E, Sandnabba NK. Exploring genetic and enviromental effects in dysphonia: a twin study. J Speech Lang Hear Res. 2009;52(1):153-63. http://dx.doi. org/10.1044/1092-4388(2008/07-0095). PMid:18664695.

6. Behlau M, Zambon F, Moreti F, Oliveira G, Barros Couto E Jr. Voice self-assessment protocols: different trends among organic and behavioral dysphonias. J Voice. 2016. In press. PMid:27210475.

7. Servilha EAM, Pena J. Tipificação de sintomas relacionados à voz e sua produção em professores identificados com ausência de alteração vocal na avaliação fonoaudiológica. Rev CEFAC. 2010;12(3):454-61. http://dx.doi.org/10.1590/S1516-18462010005000035. 
8. Choi-Cardim K, Behlau M, Zambon F. Sintomas vocais e perfil de professores em um programa de saúde vocal. Rev CEFAC. 2010;12(5):8119. http://dx.doi.org/10.1590/S1516-18462010005000075.

9. Speyer R. Effects of voice therapy: a systematic review. J Voice. 2008;22(5):565-80. http://dx.doi.org/10.1016/j.jvoice.2006.10.005. PMid:17509828.

10. Behlau M, Pontes P, Vieira VP, Yamasaki R, Madazio G. Apresentação do Programa Integral de Reabilitação Vocal para o tratamento das disfonias comportamentais. CoDAS. 2013;25(5):492-6. http://dx.doi. org/10.1590/S2317-17822013000500015. PMid:24408556.

11. Gartner-Schmidt J, Roth DF, Zullo TG, Rosen CA. Quantifying component parts of indirect and direct voice therapy related to different voice disorders. J Voice. 2013;27(2):210-6. http://dx.doi.org/10.1016/j. jvoice.2012.11.007. PMid:23352061.

12. Law T, Lee KYS, Ho FNY, Vlantis AC, Van Hasselt AC, Tong MCF. The effectiveness of group voice therapy: a group climate perspective. $\mathrm{J}$ Voice. 2012;26(2):e41-8. http://dx.doi.org/10.1016/j.jvoice.2010.12.003. PMid:21550777.

13. Almeida LNA, Fahning AKCA, Trajano FMP, Anjos UU, Almeida AAF. Group voice therapy and its effectiveness in the treatment of dysphonia: a systematic review. Rev CEFAC. 2015;17(6):2000-8. http://dx.doi.org/10.1590/1982-021620151765815.

14. Silva WJND, Lopes LW, Macedo AER, Costa DBD, Almeida AAF. Reduction of risk factors in patients with behavioral dysphonia after vocal group therapy. J Voice. 2017 Jan;31(1):123.e15-9. PMid:26897544.

15. Penteado RZ, Servilha EAM. Fonoaudiologia em saúde pública/ coletiva: compreendendo prevenção e o paradigma da promoção da saúde. Distúrb Comun. 2004;16(1):107-16.

16. Almeida AAF, Telles MAQ. Autopercepção como facilitadora de terapia vocal em grupo. Distúrb Comun. 2009;21:373-83.

17. Moreti F, Zambon F, Oliveira G, Behlau M. Cross-cultural adaptation, validation, and cutoff values of the Brazilian version of the voice symptom scale: VoiSS. J Voice. 2014;28(4):458-68. http://dx.doi. org/10.1016/j.jvoice.2013.11.009. PMid:24560004.

18. Behlau M, Madazio G, Moreti F, Oliveira G, Santos LM, Paulinelli $\mathrm{BR}$, et al. Efficiency and cutoff values of self-assessment instruments on the impact of a voice problem. J Voice. 2016 Jul;30(4):506.e9-18. PMid:26168902.

19. Cohen SM, Kim J, Roy N, Asche C, Courey M. Prevalence and causes of dysphonia in a large treatment-seeking population. Laryngoscope. 2012;122(2):343-8. http://dx.doi.org/10.1002/lary.22426. PMid:22271658.
20. Vital HRMC, Lima-Silva MFB, Almeida LNA, Almeida AAF. Sintomas vocais auditivos e sensoriais pré e pós-terapia de grupo de pacientes com disfonia. Rev CEFAC. 2016;18(5):1189-99. http:// dx.doi.org/10.1590/1982-0216201618521315.

21. Dejonckere PH, Bradley P, Clemente P, Cornut G, Crevier-Buchman L, Friedrich G, et al. A basic protocol for functional assessment of voice pathology, especially for investigating the efficacy of (phonosurgical) treatments and evaluating nem assessment techniques. Eur Arch Otorhinolaryngol. 2001;258(2):77-82. http://dx.doi.org/10.1007/ s004050000299. PMid:11307610.

22. Cielo CA, Gonçalves BFDT, Lima JPDM, Christmann MK. Afecções laríngeas, tempos máximos de fonação e capacidade vital em mulheres com disfonia organofuncional. Rev CEFAC. 2012;14(3):481-8. http:// dx.doi.org/10.1590/S1516-18462011005000126.

23. Gomes R, Nascimento EF, Araújo FC. Por que os homens buscam menos os serviços de saúde do que as mulheres? As explicações de homens com baixa escolaridade e homens com ensino superior. Cad Saude Publica. 2007;23(3):565-74. http://dx.doi.org/10.1590/S0102$311 \times 2007000300015$

24. Jones SM, Carding PN, Drinnan MJ. Exploring the relationship between severity of dysphonia and voice-related quality of life. Clin Otolaryngol. 2006;31(5):411-7. http://dx.doi.org/10.1111/j.17494486.2006.01291.x. PMid:17014451.

25. Hein R, Behlau M. Voz do especialista. Rio de Janeiro: Revinter; 2006.Perfil vocal de padres e seminaristas da igreja católica.

26. Barata LF, Madazio G, Behlau M, Brasil O. Análise vocal e laríngea na hipótese diagnóstica de nódulos e cistos Vocal and laryngeal analyses in diagnostic hypotheses of nodules and cysts. Rev Soc Bras Fonoaudiol. 2010;15(3):349-54. http://dx.doi.org/10.1590/S151680342010000300007 .

27. Ribeiro VV, Santos AB, Prestes T, Bonki E, Carnevale L, Leite APD. Autoavaliação vocal e qualidade de vida em voz de indivíduos hipertensos. Rev CEFAC. 2013;15(1):128-34. http://dx.doi.org/10.1590/ S1516-18462012005000074.

28. Pedrosa V, Pontes A, Pontes P, Behlau M, Peccin SM. The effectiveness of the comprehensive voice rehabilitation program compared with the vocal function exercises method in behavioral dysphonia: a randomized clinical trial. J Voice. 2016;30(3):377.e11. http://dx.doi.org/10.1016/j. jvoice.2015.03.013. PMid:25959424.

29. Lopes LW, Silva HF, Evangelista DS, Silva JD, Simões LB, Costa e Silva PO, et al. Relação entre os sintomas vocais, intensidade do desvio vocal e diagnóstico laríngeo em pacientes com distúrbios da voz. CoDAS. 2016;58051(4):900. http://dx.doi.org/10.1590/23171782/20162015062. 